\title{
EDITORIAL
}

\section{The Innovative Medicines Initiative (IMI): a new opportunity for scientific collaboration between academia and industry at the European level}

\author{
N. Kamel*, C. Compton\# , R. Middelveld*, T. Higenbottam* and S-E. Dahlén*
}

$\mathbf{T}$ he introduction of truly new drug treatments is dwindling to a troublesome extent in all fields of medicine, at the same time as the costs for drug development are skyrocketing. New strategies are urgently required as old schemes for drug development are failing. The largest European public-private partnership in biomedical research, the Innovative Medicines Initiative (IMI), is therefore launched. IMI is a unique pan-European research and development (R\&D) initiative with the strategic focus of strengthening the competitiveness of European Union (EU)-based biopharmaceutical industry by, amongst other activities, supporting research that aims at a faster discovery and development of safer and more effective medicines for patients.

The founding organisations of this new legal entity are the European Federation of Pharmaceutical Industries and Associations (EFPIA) [1] and the European Commission (EC). The partnership is a $€ 2$ billion joint venture, which will be set up to run over the next 10 yrs. The funding system is balanced 1:1; the EC will contribute funding and the industry will contribute in kind, for instance by providing access to specialised expertise and platform approaches. Public consortia made up of universities, hospitals, regulatory authorities, small- and medium-sized biopharmaceutical and healthcare companies (or SMEs) and patient organisations will, on a competitive basis, be able to apply for funding; if this is approved, it will be matched by equal in-kind resources from the EFPIA members.

\section{THE STRATEGIC RESEARCH AGENDA OF IMI}

The overarching objectives of IMI have been outlined in a Strategic Research Agenda (SRA) of IMI [2], which was developed over the past 3 yrs by the Research Directorate of the EC and EFPIA via numerous consultations that included stakeholders, such as academic scientists, regulatory authorities and patient groups.

\footnotetext{
*European Respiratory Society (ERS), ERS Headquarters, Lausanne, Switzerland. \#European Federation of Pharmaceutical Industries and Associations (EFPIA), EFPIA Headquarters, Brussels, Belgium.
}

STATEMENT OF INTEREST: A statement of interest for C. Compton can be found at www.erj. ersjournals.com/misc/statements.shtml. No conflict of interest is declared for IMI given its precompetitive nature.

CORRESPONDENCE: N. Kamel, European Respiratory Society, Brussels Office, Rue de Treves 4951, BE-1040, Brussels, Belgium. Fax: 32 22385361. E-mail: nadia.kamel@ersnet.org
The legal act on IMI was adopted by the Council in December 2007 and was published in the Official Journal of the European Union in February 2008 as a Council Regulation setting up the "Joint Undertaking for the Implementation of the Joint Technology Initiative on Innovative Medicines" [3], which also contains the statutes describing how IMI will be implemented. IMI was set up with its own legal entity based in Brussels, where public and private partners share governance [3].

The central aims of IMI are to enhance safety and efficacy of therapies for the major diseases, as well as to improve knowledge management and education in R\&D. Focus is on five disease areas: brain disorders, cancer, and metabolic, infectious and inflammatory diseases. Respiratory medicine is included among the inflammatory diseases, with a focus on removing the bottlenecks to identifying and progressing new therapies for patients with asthma, chronic obstructive pulmonary disease (COPD) and rhinitis. A critical aspect is the need to develop a truly collaborative effort between clusters of excellent European researchers both from academia and industry, in order to optimise translational research and help deliver novel innovative therapies. IMI is not about supporting products and individual drug-development projects; it concerns itself with tackling the knowledge and capability gaps that exist across major disease areas.

\section{BOTTLENECKS IN THE PHARMACEUTICAL R\&D PROCESS}

The biopharmaceutical industry is currently facing numerous challenges in the drug research and discovery process, which is becoming increasingly complex and expensive and has led to a decrease in the approval of a number of new therapies for marketing. On average, it takes $10-15$ yrs and many 100 millions of Euros to develop one new medicine.

Figure 1 summarises the key bottlenecks identified in the SRA [2] at different phases of drug development, ranging from the preclinical arena with a lack of predictive models for pharmacology and toxicology, through to the need for improved methods related to ongoing assessment of risk/ benefit in the market place and a truly integrated approach to knowledge management and education/training. On an individual basis, biopharmaceutical companies cannot overcome the multiple challenges that these issues present. 


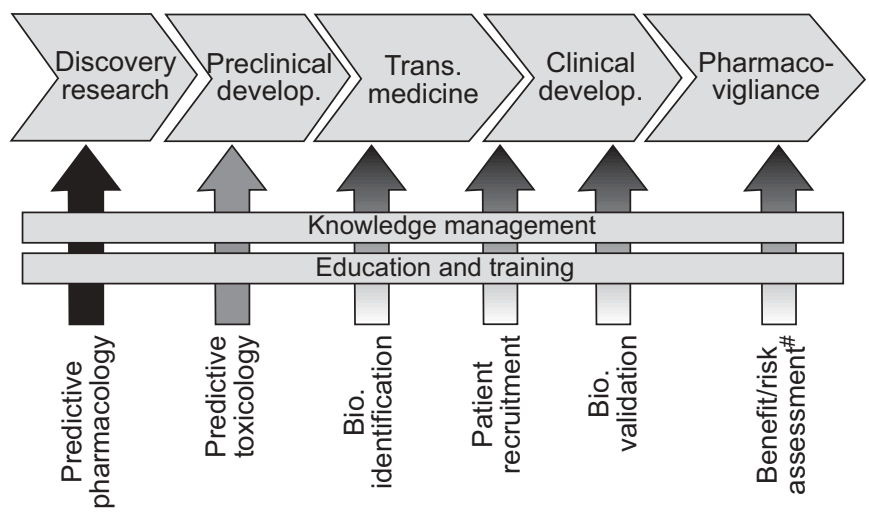

FIGURE 1. Key bottlenecks in the pharmaceutical research and development process. Develop.: development; Trans.: translational; Bio.: biomarkers. efficacy; $\square$ : safety. ${ }^{\#}$ : with regulatory authorities. Reproduced and modified from [4] with permission from the publisher.

\section{BIOPHARMACEUTICAL BOTTLENECKS IN RESPIRATORY MEDICINE}

Respiratory medicine has received priority within the area of inflammatory diseases of the SRA [2], but the areas of cancer and infectious diseases may also tackle problems of relevance to respiratory medicine. In the inflammatory disease section of the SRA [2], explicit priority is given to research in the fields of asthma, COPD and rhinitis.

The key issue is a fundamental limitation of understanding of the pathobiology of the major respiratory diseases. Despite many attractive new targets to reach with therapy, selection of those that will relieve the consequences and progression of the disease has proved to be a major impediment. Without this precise knowledge, models of disease do not reflect the human condition and early clinical studies have proved challenging in predicting large-scale effectiveness in patients. Moreover, "biomarkers" is a common theme throughout all three diseases.

The priorities for research identified in the SRA [2] therefore fall into the following three main areas. 1) Increased understanding of the natural history and disease mechanisms in the setting of disease heterogeneity, including its frequent comorbidities, and biochemical and genomic biomarkers to identify patient characteristics associated with different phenotypes/ genotypes. 2) Development of relevant preclinical and clinical models to enable translational research strategies and establish more predictive test systems. 3) Establishment of new health outcomes tools, including quality-of-life measures that reflect the patient's perspective of the disease and treatment, are sensitive to pharmacological interventions and can predict pharmacoeconomic benefit.

\section{FIRST CALL FOR RESEARCH PROPOSALS: APRIL 2008}

The responses to the first call for research proposals will be posted on several official websites and will also be communicated via the European Respiratory Society (ERS) website [5]. As indicated in figure 2, after the publication of the responses to the first call, for a 3-month period, academic research consortia may submit expressions of interest (EOIs), either alone or together with SMEs and other partners. EFPIA companies are precluded from participating in consortia

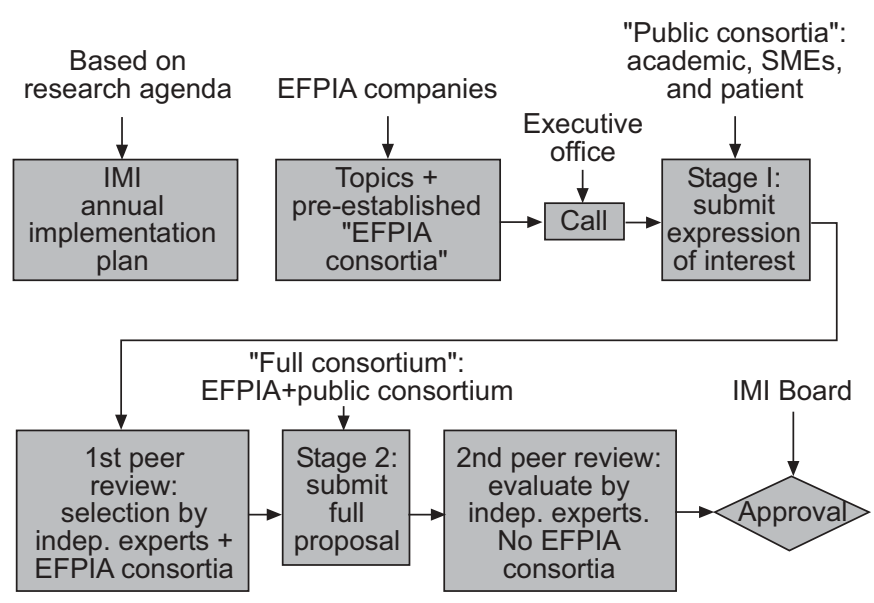

FIGURE 2. The Innovative Medicines Initiative (IMI) calls and assessment of proposals process. EFPIA: European Federation of Pharmaceutical Industries and Associations; SMEs: small and medium enterprises; indep.: independent. Reproduced and modified from [4] with permission from the publisher.

during this first stage as they will be part of the first peerreview process of the submitted EOIs. The EOIs will be assessed both by a panel of external expert reviewers and those specific EFPIA companies that have decided to invest in this particular call. A second stage will follow during which a shortlist of selected EOIs will be invited to submit a full proposal. At this stage, EFPIA companies will join particular consortia bidding for a call in a process. The call and evaluation process will be somewhat different to that in the Seventh Framework Programme (FP7) collaborative projects, as the EFPIA scientist will be engaged in the preparation of the final proposal.

\section{THE 2008 IMI RESPIRATORY CALLS ON COPD AND ASTHMA}

In the respiratory field, the overarching objective is to build a European network or "Centre of Excellence" for asthma, COPD and rhinitis throughout the life of IMI. The first IMI "efficacy" calls will represent a step towards this goal. There are two respiratory calls that it is hoped will progress in 2008, following approval by the IMI Board: one related to increasing our understanding of severe asthma, and the second to improving health outcome assessments in COPD.

\section{Call to build an EU severe asthma consortium focused on disease understanding}

The intent will be to harness efforts of already-established groups that are presently working independently. The proposed consortium would include expertise both in adults and children with severe asthma. The requirement for the consortium would include establishing diagnostic criteria, defining relevant phenotype and genotype markers, and setting up a large, longitudinal patient cohort that will act as a central platform to enable understanding of severe asthma. Ultimately, the severe asthma cohorts will enable scientific research, leading to validation of novel biomarkers and clinical measures/outcomes, and will serve as a vehicle to develop relevant translational models. 


\section{Call for patient-reported outcome tools to be used in clinical trials to evaluate treatments for COPD}

A patient-reported outcome (PRO) is any report that comes directly from a patient, without interpretation by a physician or others, about how they function or feel in relation to a health condition and its therapy. The evaluation of novel therapies for COPD poses significant challenges. Changes in clinical measures of airflow obstruction (the forced expiratory volume in one second/forced vital capacity ratio and other specific lung function tests) do not always translate to meaningful improvement in patients' symptoms or health status. Capturing the COPD patients' experience of the disease and effects of treatment is therefore an important aspect of evaluating treatments for COPD. This call would require development of a framework to understand the patients' experience of COPD, to inform strategies to measure outcomes meaningful to patients and to develop/select and validate PRO measurement tools, to use in clinical trials evaluating treatments for the disease.

\section{THE FUTURE: FURTHER CALLS AND ERS INVOLVEMENT}

IMI is keen to collect views on research areas and themes that might be developed into calls for 2009 and future years. EFPIA has been given the mandate to initiate this process while recruitment of operational staff for IMI is taking place. This is thought to involve a series of contacts with scientific experts in the field, as well as stakeholders such as patient organisations and regulatory authorities.

As a first step, the EFPIA group in respiratory medicine, which includes Almirall, AstraZeneca, Boehringer Ingelheim, Chiesi, GlaxoSmithKline, Merck, Novartis and Pfizer, decided to seek advice from the ERS. The Society's leadership then invited ERS members with recognised experience in asthma and COPD to a first Summit on IMI, which took place in Leuven (Belgium) on 9-10 January, 2008. The meeting gathered together some 30 top-level scientists in the field of COPD and asthma, and about 20 high-level R\&D investigators from the EFPIA companies. On the first day of the Summit, the company participants presented the main shortcomings and other issues in the current drug-development process. On the second day, the academic and industry investigators discussed possible remedies and strategies that would make drug development in asthma and COPD more effective. The 2-day Summit was a successful pioneering initiative for the ERS and EFPIA. A meeting report from the Summit will be published on the ERS website [4]. In future, the ERS will be following up on this Summit through the use of interactive web-based tools, such as
ERS Live sessions. The ERS is committed to keeping its members updated on the IMI and to providing opportunities for members to influence the process of the development of future calls.

\section{PERSPECTIVE}

The Innovative Medicine Initiative represents an unprecedented new era in the partnership between the European Commission, the pharmaceutical industry, academia, regulatory authorities and patients. It will certainly involve a learning process and a requirement for adjustments along the way, as with all journeys into new territory. The academic investigators must recognise that this is not simply a new European Union grant to compete for, but rather a large endeavour in which many partners will cooperate in a "pre-competitive" fashion to develop new platforms and knowledge. With an open mind and an unprejudiced attitude in all partners, there is, however, great potential that the Innovative Medicines Initiative may bring about a major boost to research in respiratory medicine within the next decade, with the overall objective of faster discovery and development of safer and more effective medicines to reduce the burden of disease in Europe.

\section{REFERENCES}

1 European Federation of Pharmaceutical Industries and Associations. www.efpia.org/DocStorage/PublicSiteAdmin/ Publications/Strategic\%20Research\%20Agenda\%20(Version\% 202).pdf

2 IMI Strategic Research Agenda http:/ /www.imi-europe.org Date last updated: September 15, 2006

3 Council Regulation (EC) No 73/2008 of 20 December 2007. Setting up a Joint Undertaking for the implementation of the Joint Technology Initiative on Innovative Medicines. CORDIS - Community Research and Development Information Service on Joint Technology Initiatives. Offical Journal of the European Union http://eur-lex.europa.eu/LexUriServ/ LexUriServ.do?uri=OJ:L:2008:030:0038.0051:EN:PDF Published: February 4, 2008.

4 The Innovative Medicines Initiative (IMI). Introducing the IMI Call Process. http:/ / www. imi-europe.org/DocStorage/ PublicSiteAdmin / Publications / IMI\%20Call\%Process $\% 20$ Overview\%20February\%202008.pdf Date last updated: February 8, 2008.

5 European Respiratory Society. www.ersnet.org 